\title{
Cardiac Tamponade from Pericardial Mesothelioma Causing Acute Renal Insufficiency in Dog
}

\author{
Sajika Seengam ${ }^{1}$, Chattida Panprom ${ }^{1}$, Pat Puncharoen ${ }^{1}$, Soontaree Petchdee ${ }^{2, *}$ \\ ${ }^{1}$ Kasetsart University Veterinary Teaching Hospital, Faculty of Veterinary Medicine, Kasetsart University, Kamphaeng Saen, Nakorn Pathom, \\ Thailand \\ ${ }^{2}$ Department of Large Animal and Wildlife Clinical Sciences, Faculty of Veterinary Medicine, Kasetsart University, Kamphaeng Saen Nakorn \\ Pathom, Thailand
}

Email address:

fvetstr@ku.ac.th (S. Petchdee)

${ }^{*}$ Corresponding author

\section{To cite this article:}

Sajika Seengam, Chattida Panprom, Pat Puncharoen, Soontaree Petchdee. Cardiac Tamponade from Pericardial Mesothelioma Causing Acute Renal Insufficiency in Dog. Cardiology and Cardiovascular Research. Vol. 3, No. 4, 2019, pp. 94-98. doi: 10.11648/j.ccr.20190304.14

Received: October 28, 2019; Accepted: November 18, 2019; Published: November 22, 2019

\begin{abstract}
Pericardial mesothelioma is a rare malignancy tumor in dog. Due to an aggressive tumor and low survival rates, mesothelioma is frequently diagnosed at an advanced stage. The objective of this clinical report is to highlight the characteristic of this malignancy tumor to help an early diagnosis and to improve the therapeutic outcomes of this neoplastic tumor. A 10-year-old crossbreed was referred to Kasetsart University Veterinary Teaching Hospital Kamphaengsaen for the evaluation of a history of abdominal distension, lethargy and weekness. During the physical examination, the dog was tachycardia and tachypnea. Ultrasonography imaging of spleen revealed an inhomogeneous appearance and thoracic radiograph showed cardiac enlargement. Electrocardigraphy examination revealed low voltage of QRS complex amplitude. A transthoracic echocardiogram showed a large pericardial effusion with cardiac tamponade and masses were visualized in pericardial sac. Pericardiocentesis was immediately performed to alleviate the cardiac tamponade and cytologic examination was performed to identify the characteristic of pericardial effusion fluid. Clinical symptoms of acute kidney injury were progressed from the second visit including azotemia, hyponatraemia, hyperkalemia and anuria. The dog died about 2 days after admission in the critical care unit. Histopathological examination of the pericardial fluid and pericardial mass confirmed the diagnosis of pericardial mesothelioma. Mesothelioma should be considered in the diagnosis of pericardial effusion in dog. An early stage detection of pericardial mesothelioma may significantly reduction in mortality and allow for more beneficial treatment.
\end{abstract}

Keywords: Cardiac Tamponade, Pericardial Tumor, Dog

\section{Introduction}

Pericardial mesothelioma is uncommon cardiac tumor in $\operatorname{dog}[1]$. Mesothelioma is a progressive malignant tumor that originated from mesodermal [2]. The common sites of mesothelioma in dog include peritoneal cavity, pleural and pericardium. Other sites have been reported such as testes and vagina [3]. Pericardial tumor can cause severe clinical symptoms such as pericardial effusion, sign of congestive heart failure, left ventricular outflow tract obstruction, arrhythmias and sudden death [4, 5]. Transthoracic echocardiography is the first-line diagnostic tool for pericardial tumors. However, echocardiography contains some limitations such as tissue characterization capability and restrict field of view in mediastinal and the right heart [6]. In human medicine, other noninvasive advance imaging modalities such as magnetic resonance imaging (MRI) and computed tomography (CT) have been applied to evaluate a cardiac tumor [7]. However, histopathological evaluation of the mass is considered to be a good diagnostic tool for a definitive diagnosis $[8,9]$. Major clinical sign of pericardial mesothelioma is recurrent pericardial effusion $[10,11]$. The effective therapeutic approach should be targeted at the etiology of tumor and controlled the effusion [12, 13]. Pericardiectomy and combination chemotherapy is the 
effective treatment for pericardial mesothelioma. However, target radiation and immune therapy are recently concerned as a future treatment of choice for uncommon tumor in dogs $[14,15]$.

\section{Case Presentation}

A 10-year-old male crossbred, weighting $25 \mathrm{~kg}$, was referred to Kasetsart Veterinary Teaching Hospital Kamphaengsaen with signs of abdominal distension and tachypnea. On physical examination, the body temperature was $37^{\circ} \mathrm{C}$, the heart rate was $150 \mathrm{bpm}$, and the respiration rate was increased $(40 / \mathrm{min})$. Systolic blood pressure was measured using a Doppler ultrasound device was $120 \mathrm{mmHg}$. Thoracic radiograph showed enlarge cardiac silhouette (Figure 1). Electrocardiography revealed low voltage of QRS complex (Figure 2), complicated with abdominal distension. During second visit, pericardiocentesis was performed for alleviating the cardiac tamponade. After 2 days admission in the hospital, the dog suffered from acute renal insufficiency, vomiting and diarrhea. Physical examination showed anorexia, depression and dehydration with heart rate 150 beats/minute, respiration 40 breathes/minute, blood pressure 100/60 mmHg.

\subsection{Second Visit and Hospitalization}

At the second visit, dog presented with lethargy, tachycardia and poor pulse quality. After routine clinical examination, dog was transferred to critical care unit was admitted for acute renal insufficiency in the second visit. The blood showed white blood cells at $16.3\left(\times 10^{3} / \mathrm{ul}\right)$, blood gas analyses showed $\mathrm{pH} 7.4$, partial pressure of carbon dioxide $19.6 \mathrm{mmHg}$, partial pressure of oxygen $52.4 \mathrm{mmHg}$, bicarbonate $13.2 \mathrm{mmol} / \mathrm{L}$, potassium $6.88 \mathrm{mmol} / \mathrm{L}$, and sodium $126.5 \mathrm{mmol} / \mathrm{L}$. Blood urea nitrogen was 99.80 $\mathrm{mmol} / \mathrm{L}$ and serum creatinine was $2.11 \mathrm{mg} \%$ (Table 1). The echocardiographic examination showed severe pericardial effusion.

\subsection{Clinical Evaluations}

Dog's owner was provided consent form to approve the therapeutic procedure. Routine laboratory evaluations were performed included hematology, serum biochemistry and electrolyte levels analysis as shown in Table 1. Pericardiocentesis was performed and $400 \mathrm{ml}$ of serosanguineous pericardial exudate was removed from the pericardial cavity to alleviate a sign of cardiac tamponade. After pericardiocentesis, no effusion fluid was observed in the pericardial sac. A full cardiac examination was performed as shown in Table 2, including Electrocardiography, 2Dimensional, M-Mode and color flow Doppler echocardiography (vivid s5, USA). Echocardiography was performed in parasternal long, short axis views and apical four-chamber view in right and left parasternal position with no sedation. Echocardiographic images were captured and stored for offline analysis. Left ventricular wall structure and function were calculated by measuring the images from twodimensional and M-mode planes (Figure 3).

\subsection{Cytology and Histopathological Analysis}

Cytology from a transudative pleural effusion showed central round nuclei with light purple cytoplasm of mesothelioma cells (Figure 4). Histopathology of the isolated tumor from pericardium demonstrated tubules like structures cells which confirm pericardial mesothelioma (Figure 5).

Figures:
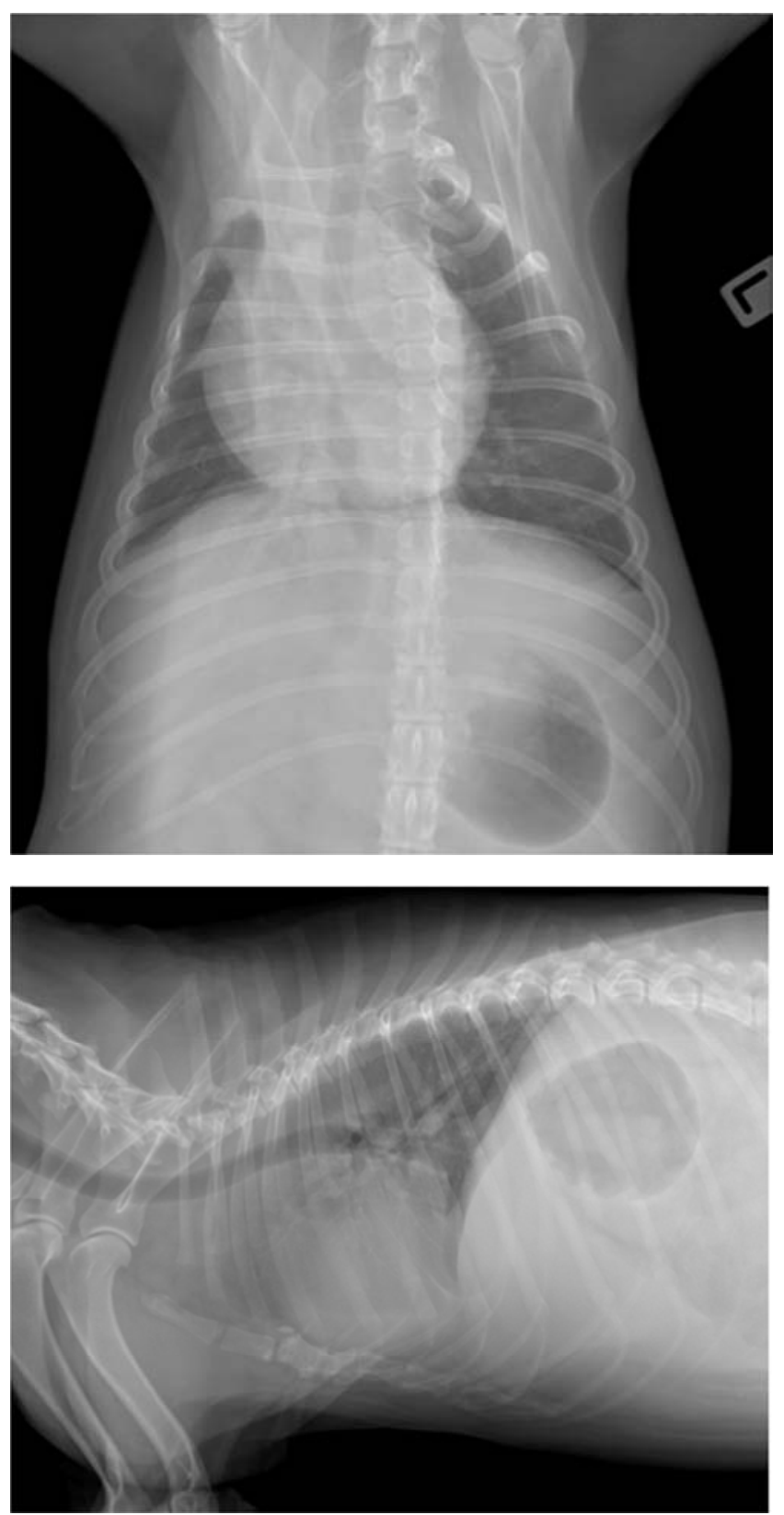

Figure 1. Thoracic radiographs showing enlarge cardiac silhouette on ventrodorsal and lateral view. 

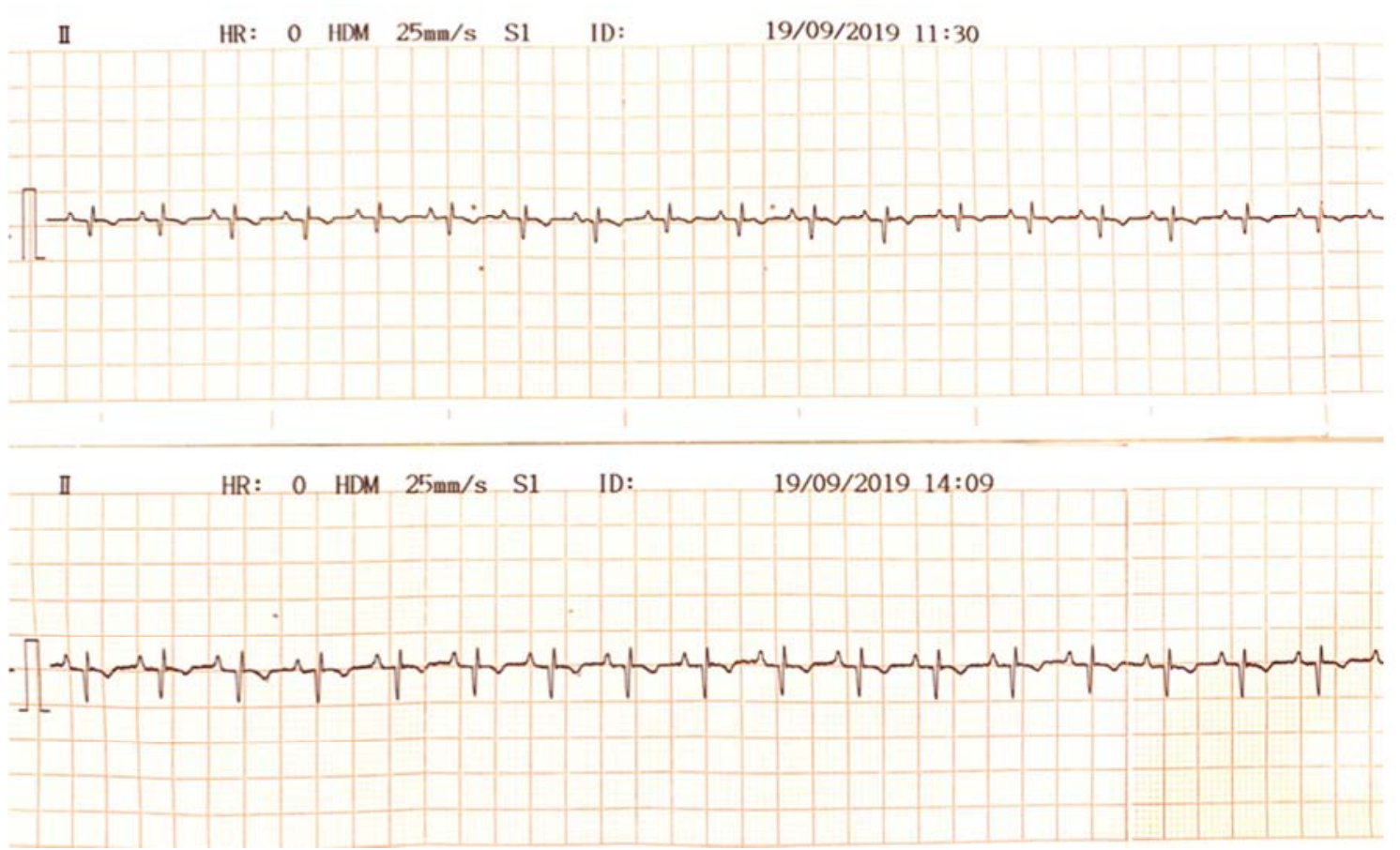

Figure 2. Electrocardiography before (a) and after pericardiocentesis (b).
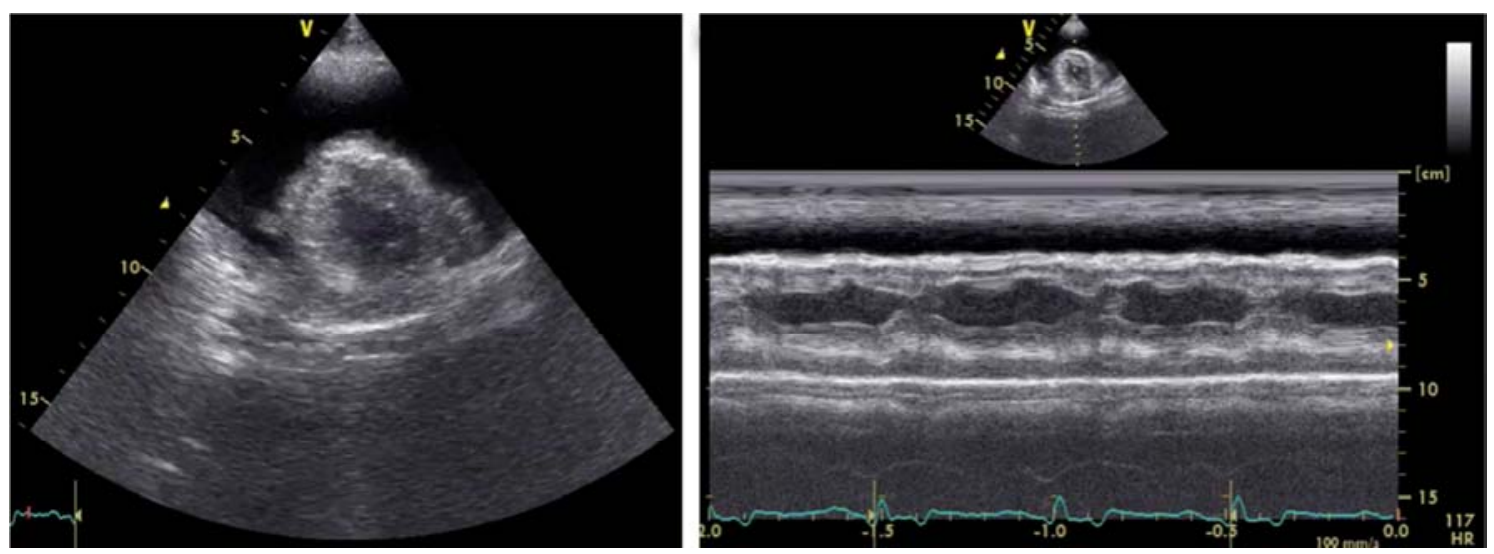

Figure 3. Echocardiographic of transverse image of left ventricle (left) and M mode of the left ventricle (right) during diastole. Images showing tumors in pericardial sac, collapse of right atrium and the marked pericardial effusion.

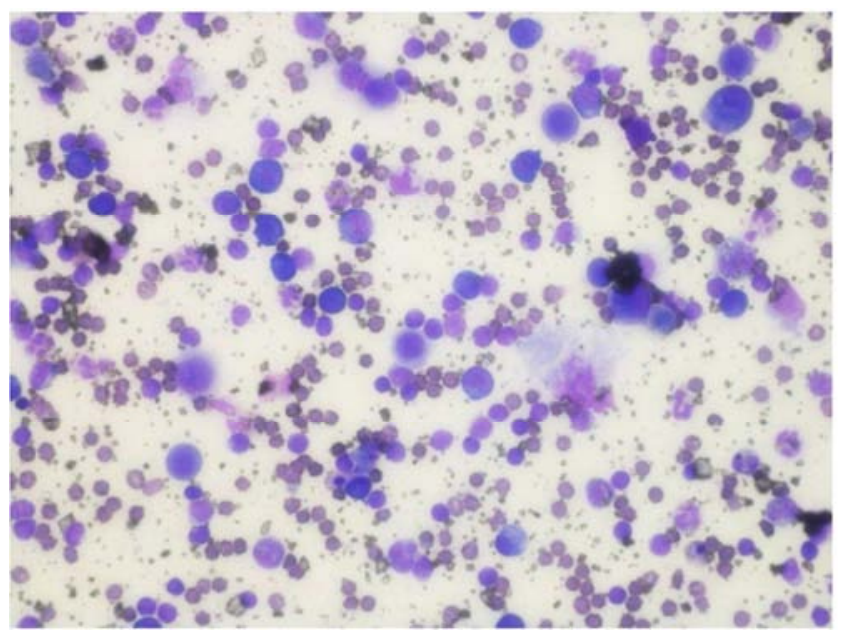

Figure 4. Cytology from a pleural effusion in a dog with pericardial mesothelioma (H\&E stain, $x$ 400).

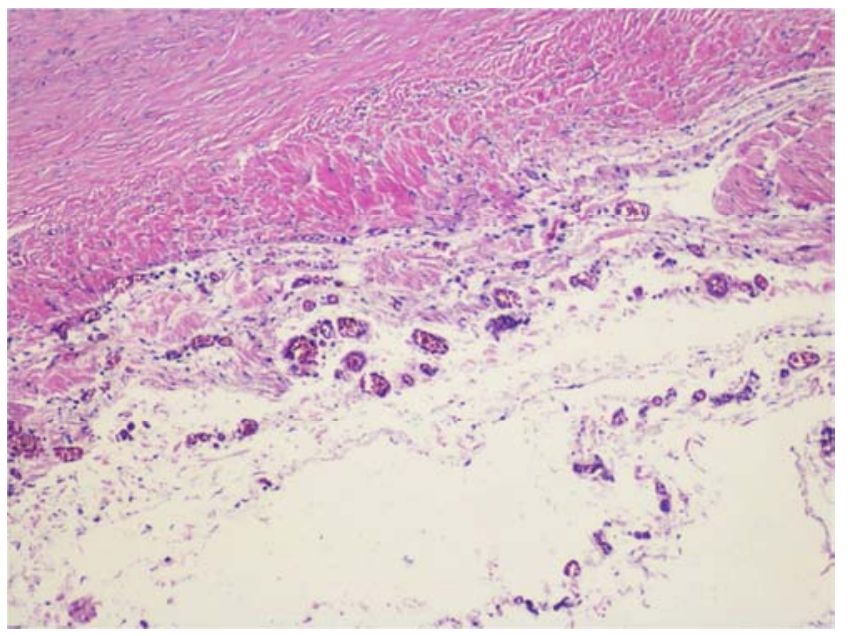

Figure 5. Analysis of neoplastic mass in pericardium. H\&E image (x40) revealed diffuse infiltration of tubules like structure cells and hemorrhagic foci are observed throughout the neoplastic lesion. 
Table 1. Blood profiles at first visit and during admission of second visit.

\begin{tabular}{llll}
\hline & First visit & During admission & Second day admission \\
\cline { 3 - 4 } & & First day admission & 21.45 \\
$\mathrm{WBC}\left(\mathrm{x} 10^{3} / \mathrm{ul}\right)$ & 24.90 & 16.31 & 4.54 \\
$\mathrm{RBC}\left(\mathrm{x} 10^{6} / \mathrm{ul}\right)$ & 6.55 & 5.59 & 9.9 \\
$\mathrm{HGB}(\mathrm{g} / \mathrm{dL})$ & 13.60 & 11.80 & 29 \\
$\mathrm{HCT}(\%)$ & 39.80 & 31.70 & 604 \\
$\mathrm{PLT}\left(\mathrm{x} 10^{3} / \mathrm{ul}\right)$ & 389 & 371 & 10.40 \\
$\mathrm{Creatinine}(\mathrm{mg} \%)$ & 0.87 & 2.11 & 298.00 \\
$\mathrm{BUN}(\mathrm{mg} \%)$ & 20.60 & 99.80 & 120 \\
$\mathrm{ALT}(\mathrm{IU} / \mathrm{L})$ & 136 & 120 & 2.3 \\
$\mathrm{Albumin}(\mathrm{gm} \%)$ & 2.7 & 2.10 & 7.43 \\
$\mathrm{pH}$ & 7.4 & 7.40 & 23.5 \\
$\mathrm{pCO}(\mathrm{mmHg})$ & 29 & 19.60 & 50.6 \\
$\mathrm{pO}_{2}(\mathrm{mmHg})$ & 54 & 52.40 & 134.4 \\
$\mathrm{Na}^{+}(\mathrm{mmol} / \mathrm{L})$ & 145 & 126.5 & 6.48 \\
$\mathrm{~K}^{+}(\mathrm{mmol} / \mathrm{L})$ & 4.5 & 6.88 & \\
\hline
\end{tabular}

$\mathrm{WBC}=$ White blood cell $; \mathrm{RBC}=$ Red blood cell $\mathrm{HGB}=$ hemoglobin; $\mathrm{HCT}=$ hematocrit PLT $=$ Platelet; $\mathrm{ALT}=$ Alanine aminotransferase

Table 2. Echocardiagraphic parameters in dog with cardiac hemangiosarcoma.

\begin{tabular}{lll}
\hline Parameters & Patient & Reference range \\
\hline IVSd $(\mathrm{cm})$ & 1.1 & $0.7-1.4$ \\
LVIDd $(\mathrm{cm})$ & 4.8 & $3.7-5.4$ \\
LVPWd $(\mathrm{cm})$ & 1.3 & $0.7-1.4$ \\
IVSs $(\mathrm{cm})$ & 1.5 & $1.0-1.9$ \\
LVIDs $(\mathrm{cm})$ & 3.2 & $2.3-4.0$ \\
LVPWs $(\mathrm{cm})$ & 1.5 & $1.1-2.0$ \\
EF $(\%)$ & 61 & $49-81$ \\
FS $(\%)$ & 33 & $25-45$ \\
LA/Ao Ratio & 1.21 & $1.0-1.5$ \\
EPSS $(\mathrm{cm})$ & 0.6 & $0.4-0.75$ \\
\hline
\end{tabular}

IVSd; diastolic interventricular septum thickness, IVSs; systolic interventricular septum thickness, LVIDd; left ventricular end diastolic diameter, LVIDs; left ventricular end systolic diameter, LVPWd; left ventricular wall diastolic thickness, and LVPWs; left ventricular wall systolic thickness. The left ventricular ejection fraction (EF); EPSS, E point of septum separation.

\section{Discussion}

Complete blood counts and serum biochemistry evaluations were performed, hematology results were compatible with thrombocytopenia and leukocytosis. Signs of cardiac tamponade was observed after pericardiocentesis and the electrocardiography showed sinus tachycardia rhythm. The pericardial effusion fluid contained red blood cells and reactive mesothelial cells with $\mathrm{pH}$ 8.0. After the second visit, the blood profiles showed gradual impairment of renal function as shown in Table 1. Abdominal ultrasonography showed ascites and inhomogeneous appearance of liver and spleen. No metastasis of other abdominal organs such as liver and kidney was visualized. Transthoracic echocardiography revealed of tumor masses on pericardium sac and marked pericardial effusion. A large pericardial effusion can cause a diminished cardiac output and neoplasia can metastasize to other organs such as aortic body or inside the cardiac chambers. However, there is no thrombus and ventricular outflow tract obstruction was observed in this case (Figure 3). In this present study, echocardiography result was highly suspicious for cardiac tumor. According to our knowledge, primary pericardial mesothelioma represents one of the rare cases in dog. However, appropriate diagnosis and treatment can lead to the excellent outcomes even if the initial severe presentation. As in this case, diagnostic imaging such as radiography, ultrasonography and echocardiography were used for initial diagnosis. However, limitations in the cytological and histopathology diagnosis still exist, pericardiectomy and pericardial biopsy might be necessary for diagnosis and resulted in the successful start of therapy. In our case, the dog was presented again 1 week after the first visit with poor appearance signs such as decreased appetite, weight loss and lethargy, systemic signs such as vomit and diarrhea were seen when the gastrointestinal tract was affected by the mesothelioma. Heart failure and acute renal insufficiency were developed that showed an unsuccessful treatment of pericardial mesothelioma in our case.

\section{Conclusions}

The prognosis for mesothelioma is guard to poor and the survival time is low due to the rapid progression and the difficulty of making diagnosis in the early stage. Timely diagnosis of pericardial mesothelioma and chemotherapy may provide the best result for treatment of uncommon primary pericardial mesothelioma in dogs.

\section{Acknowledgements}

Authors are grateful to Kasetsart University Veterinary Teaching Hospital, Kamphaeng Saen for providing the necessary information. 


\section{Author Contribution}

Sajika Seengam, Chattida Panprom, Pat Puncharoen prepared and interpreted the results and Soontaree Petchdee drafted, critical revised and approved a manuscript.

\section{References}

[1] Closa, J. M., A. Font, J. Mascort, (1999). Pericardial mesothelioma in a dog: long-term survival after pericardiectomy in combination with chemotherapy. J Small Anim Pract. 40, 383-386.Feng, X., L. Zhao, G. Han, M. Khalil, F. Green, T. Ogilvie. (2012). A case report of an extremely rare and aggressive tumor: primary malignant pericardial mesothelioma. Rare Tumors. 2, e21.

[3] Day, M. J., V. M. Lucke, H. Pearson, (1995). A review of pathological diagnoses made from 87 canine splenic biopsies. J Small Anim Prac. 36, 426-433.

[4] D'Angelo, A. R., G. Francesco, G. R. Di Quaglione, G. Marruchella, (2014). Sclerosing peritoneal mesothelioma in a dog: histopathological, histochemical, and immunohistochemical investigations. Vet Ital. 50, 301-305.

[5] Eren, N. T. and A. R. Akar, (2002). Primary pericardial mesothelioma, Current Treatment Options in Oncology. 3 (5), 369-373.

[6] Kong, L., Z. Li, J. Wang, X Lv. (2018). Echocardiographic characteristics of primary malignant pericardial mesothelioma and outcomes analysis: a retrospective study. Cardiovasc Ultrasound. 16 (7), 1-8.

[7] Smith, W. H., D. J. Beacock, A. J. Goddard, (2001). Magnetic resonance evaluation of the pericardium. $\mathrm{Br} \mathrm{J}$ Radiol. 74
(880), 384-392.

[8] Ikede, B. O., A. Zubaidy, C. W. Gill, (1980). Pericardial mesothelioma with cardiac tamponade in a dog. Vet Pathol. $17,496-500$.

[9] MacGregor, J. M., M. L. E. Faria, A. S. Moore, A. H. Tobias, D. J. Brown, H. S. A. de Morais, (2005). Cardiac lymphoma and pericardial effusion in dogs: 12 cases (1994-2004). J. Am. Vet. Med. Assoc. 227, 1449-1453.

[10] Imazio, M., B. M. Mayosi, A. Brucato, G. Markel, R. Trinchero, D. H. Spodick, Y. Adler, (2010). Triage and management of pericardial effusion. J Cardiovasc Med. 11, 928-935.

[11] Sagrista'-Sauleda, J, A, S, Merce, J., Soler-Soler, (2011). Diagnosis and management of pericardial effusion. World $\mathrm{J}$ Cardiol. 3, 135-143.

[12] Garrett, L. D., Mesothelioma. In: Withrow S. J., D. M. Vail, R. L. Page, editors. Withrow and MacEwen's Small Animal Clinical Oncology, 5th ed. Amsterdam: Elsevier (2013). p. 696-700.

[13] Reggeti, F., B. Brisson, K. Ruotsalo, E. Southorn, D. Bienzle, (2005). Invasive epithelial mesothelioma in a dog. Vet Pathol. $42,77-81$.

[14] Petrich, A., S. I. Cho, and H. Billett, (2011). Primary cardiac lymphoma: an analysis of presentation, treatment, and outcome patterns. Cancer. 117, 581-589.

[15] Tong, L. J., S. L. Bennett, D. J. Thompson, S. L. Adsett, and R. E. Shiel, (2015). Right-sided congestive heart failure in a dog because of a primary intracavitary myocardial lymphoma. Aust. Vet. J. 93, 67-71. 